ORIGINAL ARTICLE

\title{
Introduction of HIV post-exposure prophylaxis for sexually abused children in Malawi
}

\section{J C Ellis, S Ahmad, E M Molyneux}

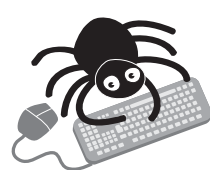

The appendices are available on the $A D C$ website (www.archdischild.com/ supplemental)

See end of article for authors' affiliations

....................

Correspondence to: Dr J C Ellis, Department of Paediatrics, College of Medicine, Private Bag 360, Blantyre 3, Malawi; janeellis123@yahoo.co.uk

Accepted

5 September 2005

Published Online First

20 September 2005
Arch Dis Child 2005;90:1297-1299. doi: 10.1136/adc.2005.080432

Aims: To improve the care of children who are victims of child sexual abuse (CSA) by routinely assessing eligibility for HIV post-exposure prophylaxis (PEP) and to investigate the feasibility, safety, and efficacy of such treatment started in a paediatric emergency department in Malawi.

Methods: Children presenting to the Queen Elizabeth Central Hospital, Blantyre between 1 January 2004 and 31 December 2004 with a history of alleged CSA were assessed for eligibility for HIV PEP and followed prospectively for six months.

Results: A total of 64 children presented with a history of alleged CSA in the 12 month period; 17 were offered PEP. The remainder were not offered PEP because of absence of physical signs of abuse $(n=20)$, delay in presentation beyond 72 hours from assault $(n=11)$, repeated sexual abuse in the preceding six months $(n=15)$, and HIV infection found on initial testing $(n=1)$. No family refused an HIV test. No side effects due to antiretroviral therapy were reported. Of the 17 children commenced on PEP, 11 returned for review after one month, seven returned at three months, and two of 15 returned at six months post-assault. None have seroconverted.

Conclusions: In a resource-poor setting with a high HIV prevalence, HIV PEP following CSA is acceptable, safe, and feasible. HIV PEP should be incorporated in to national guidelines in countries with a high community prevalence of HIV infection.
$\mathrm{H}$ IV post-exposure prophylaxis (PEP) is a form of secondary HIV prevention that is used to reduce the risk of HIV transmission. It is well established as a treatment for occupational exposure to HIV infection when healthcare workers are exposed to HIV through their work. ${ }^{12}$ In such settings it has been shown to reduce risk of transmission rate by $81 \%{ }^{3}$

Increasingly HIV PEP is prescribed in non-occupational settings. ${ }^{4}$ These include sexual assault, consensual unprotected sex, injecting drug use, and needle-stick or sharp object injuries in persons other than healthcare professionals. Recommendations for non-occupational PEP are available in both Europe and the USA. ${ }^{15-7}$

The transmission rate for HIV infection after a single sexual encounter between two consenting adults is low, estimated to be $0.01-0.2 \%{ }^{8}$ However, in child sexual abuse, because of the increased incidence of violence and trauma, the immature, thin vaginal epithelium, and cervical ectopy, the risk of transmission is likely to be much higher. ${ }^{9}$

In countries in Africa where the community prevalence of HIV infection is high, the risk of transmission of HIV infection through child sexual abuse is likely to be further increased. Although data on the actual risk is difficult to obtain, several studies offer information. In Togo, one child of 33 investigated for sexually transmitted infection (STI) following sexual abuse was found to have been infected with HIV. ${ }^{10}$ In a study from Nigeria, $4.3 \%$ of children with AIDS acquired HIV infection through sexual abuse. ${ }^{11}$ In Cameroon, an alarming 24 of 71 victims (37.5\%) of child sexual abuse acquired HIV infection secondary to the abuse. ${ }^{12}$

Because of the increasing numbers of sexually abused children presenting to our department over the preceding three years and concerns regarding the risk of transmission of
HIV infection in a high prevalence setting, we felt a real and urgent need to improve the care given to these children by introducing HIV PEP.

Here we review our experience and results from a paediatric emergency department in Malawi following a 12 month period of routinely offering HIV PEP to all eligible children following child sexual abuse to potentially prevent acquisition of HIV infection using a locally devised protocol.

\section{METHODS}

\section{Setting}

Malawi is a country in central-southern Africa with a population of 12 million. The prevalence rate of HIV infection in adults ( $15-49$ years) is $14.4 \% .^{13}$ In the Malawi National Guidelines for Antiretroviral Therapy the use of PEP for sexual assault is mentioned, but no specific protocol for management is documented and it has not been implemented. ${ }^{14}$

Blantyre is the major urban centre in the south of the country with a population of 650000 , of which $44 \%$ are under 14 years old. Queen Elizabeth Central Hospital (QECH) in Blantyre is the tertiary level referral hospital for the southern region of Malawi. The paediatric emergency department sees 75000 children per year.

In the QECH the care of victims of child sexual abuse is a consultant led service. During every assessment demographics are collected together with details and timing of the assault. A full medical history is obtained and a complete examination performed. Since January 2002 findings have been documented on a standard clinical assessment form

Abbreviations: ART, antiretroviral therapy; CSA, child sexual abuse; PEP, post-exposure prophylaxis; STI, sexually transmitted infection 
(Appendix 1; see $A D C$ website: www.archdischild.com/ supplemental).

Prior to 2004 the only feasible options for children who were victims of CSA, considering the resources available, were antibiotic treatment for prophylaxis against bacterial STI and the offer of an appointment for HIV testing three months after the assault. Few of these children returned for testing and no formal data were collected.

\section{Methods}

From 1 January 2004 each child presenting with a history of alleged sexual abuse was assessed for eligibility for HIV PEP following a written protocol (Appendix 2; see $A D C$ website: www.archdischild.com/supplemental). The child was considered eligible if he or she was seen less than 72 hours after the abuse, had not been previously assaulted in the preceding six months, and there was physical evidence of sexual penetration. In addition we asked if the HIV status of the assailant was known or could be ascertained.

If the child was eligible for PEP the guardian (and child if he or she had sufficient understanding and maturity) was counselled and an HIV test was performed (using Determine HIV-1/2 rapid test, Abbot Laboratories, USA). If the child was HIV uninfected, PEP was offered and commenced immediately.

If the child was found to be HIV infected, the guardian was counselled and the child referred to the appropriate paediatric clinic.

The antiretroviral therapy (ART) used for PEP was zidovudine and lamivudine, given for a period of 28 days (doses in Appendix 2; see $A D C$ website: www.archdischild. com/supplemental).

A child commenced on HIV PEP was reviewed at one month to assess adherence to treatment, any side effects of ART, and to identify any other physical, social, or emotional problems. The child was seen again at three and six months post-assault, for repeat HIV testing.

If the child was not eligible for HIV PEP, an appointment was arranged for three months to repeat the HIV test and review the child.

Under present rules local ethics committee approval was not deemed necessary because this treatment is recognised as standard practice in resource-rich settings following known or high risk HIV exposure.

\section{RESULTS}

In 2004 the number of children presenting to our department following alleged CSA was 64. The perpetrator was a family member in six cases $(9.4 \%)$, a known individual (neighbour, household servant, teacher) in 40 cases $(62.5 \%)$, and an unknown person in $17(28.1 \%)$. One victim was male and 63 were female. The median age was 83 months (range $22-180$ months).

Of the 64 children seen, $44(68.8 \%)$ had physical signs consistent with penetrative sexual abuse. Eleven children $(17.2 \%)$ presented more than 72 hours after the abuse. Fifteen children (23.4\%) had been assaulted previously in the preceding six months. In no case was the HIV status of the assailant known or was there the possibility of tracing the person. Therefore 18 children $(28.2 \%)$ were considered eligible for PEP. None of the families refused HIV testing. One child was found to be HIV infected on initial testing. The remaining $17(26.6 \%)$ were offered HIV PEP and all consented to treatment. The children started on PEP presented an average of 25 hours (range 8-68 hours) after the assault.

Of the 17 children started on HIV PEP, 11 (64.7\%) returned for review after one month of treatment. None of the children had side effects from the ART. None had evidence of STI.
Seven of 17 (41.2\%) and two of 15 (13.3\%) have returned for the three and six months appointments respectively. None have seroconverted.

Those children not eligible for HIV PEP were offered an appointment at three months for review of the child and repeat HIV testing. Of the 46 children, only three presented for review and none of these had seroconverted.

\section{DISCUSSION}

The number of children presenting to our department is likely to represent a small fraction of the total number who have been sexually abused. In addition to the problems of case identification experienced elsewhere, in Malawi families rarely seek medical help first unless there is evidence of severe injury. Most go first to the police and from there are referred to the hospital for a medical report. Hence if the family do not inform the police they are unlikely to present for medical assistance.

We identified a significant number of children who had been repeatedly abused over a period of time. These children were not eligible for PEP because of the possibility of the child having already acquired HIV infection and being in the "window period" prior to seroconversion.

Ideally PEP should be started as soon as possible after HIV exposure and certainly within 72 hours. Unfortunately a large number of children were excluded from PEP due to a delay in presentation. The causes of the delay are multiple. The child may not immediately disclose the assault, the family may not believe the child or decide not to seek treatment urgently, transport may be problematic, and legal notification usually takes precedent over medical assessment.

We acknowledge that by excluding children with no physical signs of abuse we are excluding children who have been abused as it is accepted that sexual penetration can occur without signs on physical examination. However if no trauma is sustained, the risk of acquiring HIV infection from one episode of sexual assault is much reduced. In this particular situation we therefore felt that the risks of treatment outweighed the benefits.

In the UK "National guideline for the management of sexually transmitted infections in children and young people" it states that "inadequate information on the risks and benefits of PEP for HIV following abuse or assault in young people makes routine recommendations impossible ${ }^{\prime \prime} .{ }^{15}$ The Children's HIV Association of the UK and Ireland (CHIVA) classes child sexual abuse as only a "moderate risk" for HIV acquisition and therefore states that the risk of PEP probably outweighs the benefits. ${ }^{16}$

However, in a high HIV prevalence setting such as Malawi we feel that the assumption should be made that the assailant is HIV infected unless known not to be. We feel that all children who have been sexually abused should be assessed for eligibility for HIV PEP and if there are no contraindications this should be offered as routine. This practice is recommended in South Africa, a country which is less resource constrained than Malawi, but with a high level of sexual violence and HIV infection. ${ }^{17}$

We found that by discussing the possibility of HIV exposure at the time of presentation, being able to perform an immediate HIV test and initiating HIV PEP for eligible children at the initial consultation proved very efficient. This coordinated approach meant there was no need to refer to other departments or members of staff, and there was less possibility of the child missing the opportunity to access PEP.

Although our return rate at one month was acceptable, the subsequent follow up rate was low. This has been found in other studies. ${ }^{18}{ }^{19}$ The reasons are multiple and complex and may be carer or child related. Carer issues may include lack of understanding of the need for follow up, or poverty and 


\section{What is already known on this topic}

- In addition to the recognised consequences of CSA, in areas of high HIV prevalence there is the risk of transmission of HIV infection to the child

- HIV PEP can prevent transmission of HIV infection but there is limited data on its use in sexually abused children in a resource-poor, high HIV prevalence setting

problems of access to health care. Child related reasons for failing to return may include adverse drug reactions or traumatic associations after the assault.

Tests to monitor for side effects of ART are not routinely available in our hospital. However, none of the children receiving ART reported any symptoms of side effects. Other studies have reported a high rate of stopping medication due to side effects. ${ }^{19-21}$ We followed Malawi national guidelines ${ }^{14}$ using a two drug regime without a protease inhibitor and therefore would expect less side effects.

For those children not eligible for HIV PEP the return rate was very poor, as was also found for sexually abused children prior to the introduction of the PEP treatment. The reasons are unclear but may include a fear of seroconversion. Because of the low return rate it is impossible to comment on the risk of seroconversion without HIV PEP.

We aim to make our service to abused children consultant led. We acknowledge that it is a specialised field within paediatrics that needs sensitivity and experience. We initiated HIV PEP as a pragmatic approach to tackle one tragic complication of CSA, but this is just one aspect of the management of children who have been sexually abused. Ideally these children should receive medical, social, and psychological care. However, in Malawi there is no formal procedure for child protection. There is no provision for emergency contraception or psychological support. Police and most first-line medical staff are not trained in the management of victims of CSA.

Since completing this policy of assessing victims of CSA for eligibility for HIV PEP we have made our protocol known nationally in Malawi. We feel that this treatment should be made available to all Malawian children who are sexually abused. However, a limiting factor for HIV PEP being offered more widely will be identifying clinicians with experience in dealing with CSA, who are able to handle these children appropriately and recognise the often subtle physical signs of abuse.

\section{Conclusions and recommendations}

Child sexual abuse affects every society and the number of affected children is increasing. HIV PEP is an important part of the care of sexually abused children in a high prevalence setting and has been shown to be safe, acceptable, and feasible. We recommend that it is routinely offered to all eligible children as an opportunity to prevent transmission of HIV infection. HIV PEP following sexual abuse should be incorporated into national guidelines in countries such as Malawi with high prevalence of HIV infection.

\section{ACKNOWLEDGEMENTS}

We would like to thank Dr Joan Robson (Consultant Paediatrician, Alder Hey Children's Hospital, Liverpool) who designed the clinical assessment form for children presenting with alleged CSA. We would also like to thank MSF-Thyolo for providing a supply of ART until the hospital pharmacy supply was available.
What this study adds

- HIV PEP following child sexual abuse is acceptable, safe, and feasible in our setting

- Integration of HIV PEP into national guidelines in areas of high HIV prevalence is recommended

\section{Authors' affiliations}

J C Ellis, S Ahmad, E M Molyneux, Department of Paediatrics, College of Medicine, University of Malawi, Blantyre, Malawi

Competing interests: none

\section{REFERENCES}

1 Department of Health. HIV post exposure prophylaxis: guidance from the UK Chief Medical Officer's Expert Advisory Group on AIDS. London, Department of Health, February 2004 (www.advisorybodies.doh.gov.uk/eaga/PDFS/ prophylaxisguidancefeb04.pdf).

2 CDC. Updated U.S. Public Health Service guidelines for the management of occupational exposures to HBV, HCV, and HIV and recommendations for postexposure prophylaxis. MMWR 2001;50(RR-11):1-42.

3 Cardo DM, Culver DH, Ciesielski CA, et al. A case-control study of HIV seroconversion in health care workers after percutaneous exposure. Centre for Disease Control and Prevention Needlestick Surveillance Group. N Engl J Med 1997; 337:1485-90.

4 Giele CM, Maw R, Carne CA, et al. British Co-operative Clinical Group of the Medical Society for the Study of Venereal Diseases. Post-exposure prophylaxis for non-occupational exposure to HIV: current clinical practice and opinions in the UK, Sex Transm Inf 2002;78:130-2.

5 Havens PL, Committee on Pediatric AIDS. Post exposure prophylaxis in children and adolescents for nonoccupational exposure to human immunodeficiency virus. Pediatrics 2003;111:1475-89.

6 Almeda J, Casabona J, Simon B, et al. Proposed recommendations for the management of HIV post-exposure prophylaxis after sexual, injecting drug or other exposures in Europe. Eurosurveillance 2004;9:35-40

7 Centers for Disease Control and Prevention. Antiretroviral postexposure prophylaxis after sexual, injection-drug use, or other nonoccupational exposure to HIV in the United States. MMWR Recomm Rep, 2005 (www.cdc.gov/mmwr/mmwr_rr.html).

8 Gray RH, Wawer MJ, Brookmeyer R, et al. Probability of HIV-1 transmission per coital act in monogamous, heterosexual, HIV-1-discordant couples in Rakai, Uganda. Lancet 2001;357:1149-53.

9 Gutman LT, Herman-Giddens ME, McKinney RE Jr. Pediatric acquired immunodeficiency syndrome. Barriers to recognising the role of child sexual abuse. Am J Dis Child 1993;147:775-80.

10 Pitche $\mathbf{P}$, Kombate K, Gbadoe AD, et al. Sexually transmitted diseases in young children in Lome (Togo). Role of sexual abuse. Arch Pediatr 2001;8:25-31

11 Angyo IA, Okpeh ES, Onah J. Paediatric AIDS in Jos, Nigeria. West Afr J Med 1998;17:268-72.

12 Menick DM, Ngoh F. Seroprevalence of HIV infection in sexually abused children in Cameroon. Med Trop 2003;63:155-8.

13 National AIDS Commission. National estimate of HIV/AIDS in Malawi in 2003, Lilongwe, Malawi, 2003.

14 Ministry of Health, Malawi. Treatment of AIDS: Guidelines for the use of antiretroviral therapy in Malawi, 1st edn. October 2003.

15 Thomas A, Forster G, Robinson A, et al. National guideline for the management of sexually transmitted infections in children and young people. Arch Dis Child 2003;88:303-11.

16 Tudor-Williams G. Post-exposure prophylaxis (PEP) guidelines for children exposed to blood-borne viruses. June 2003 (www.bhiva.org/ chiva/).

17 Kistner U. Rape and post exposure prophylaxis in South Africa: a review 2003. Centre for AIDS Development, Research and Evaluation (CADRE), South Africa

18 Wiebe ER, Comay SE, McGregor M, et al. Offering HIV prophylaxis to people who have been sexually assaulted: 16 months experience in a sexual assault service. CMAJ 2000;162:641-5.

19 Babl FE, Cooper ER, Damon B, et al. HIV postexposure prophylaxis for children and adolescents. Am J Emerg Med 2000;18:282-7.

20 Kahn JO, Martin JN, Roland ME, et al. Feasibility of postexposure prophylaxis (PEP) against human immunodeficiency virus infection after sexual or injection drug use exposure: the San Francisco PEP Study. $J$ Infect Dis $2001 ; 183: 707-14$.

21 Limb S, Kawsar M, Forster GE. HIV post-exposure prophylaxis after sexual assault: the experience of a sexual assault service in London. Int J STD AIDS 2002; 13:602-5. 